\title{
A Study of Optical Uniformity of Lithium Niobate and Paratellurite Crystals by the Method of Conoscopy
}

\author{
A.I. Kolesnikov ${ }^{a}$, S.A. Tretiakov ${ }^{a}$, R.M. Grechishinin ${ }^{a}$, K.A. Morozova ${ }^{a}$, \\ K.B. YUShKOV ${ }^{b}$, V.YA. MOLChANOV ${ }^{b}$ And B.B.J. Linde ${ }^{c}$ \\ ${ }^{a}$ Tver State University, 170100 Tver, Russia \\ ${ }^{b}$ National University of Science and Technology "MISIS", 119049 Moscow, Russia \\ ${ }^{c}$ Institute of Experimental Physics, University of Gdańsk, 80-952 Gdańsk, Poland
}

\begin{abstract}
An exact equation of the isochromatic fringes of uniaxial crystals derived without usually adopted simplifications is presented. It enables to calculate the optical conoscopic figures for ideal crystals with different angular orientations of the optical axis with respect to the normal to the crystal surface. Conoscopic figures of large-size single crystals of lithium niobate and paratellurite were examined experimentally making use of a laser-based setup. The conoscopic figure evolution in operating acousto-optic devices was studied.
\end{abstract}

DOI: $10.12693 /$ APhysPolA.127.84

PACS: 42.79.Jq, 65.40.-b

\section{Introduction}

The method of conoscopy serves as a well-known approach for the characterization of optically anisotropic crystals. It permits to determine such characteristics of a crystal as category, optical sign, angle between optical axes of biaxial materials, orientation of the normal of a sample with respect to the crystallographic directions [1-3]. This method enables to reveal anomalous uniaxiality in cubic crystals or biaxiality in uniaxial ones. In addition, it makes possible to estimate the mechanical stresses causing changes in the optical indicatrix $[3,4]$. The recent increasing interest to the method of conoscopy is related to its new potential capabilities of application in the study of optical effects in liquid crystals, nanostructures, in mineralogy, biology, holography [5-8].

Up to date the prevailing experimental implementation of the conoscopic method is based on the exploitation of polarized microscopes which are of limited use with regard to large-size objects. Small focal lengths and angular apertures of optical microscope set limits to the number of observable isochromates. In the last decades the researchers started to use lasers for conoscopic observations [9-11]. Laser ray is transformed into a bundle of conical shape producing the conoscopic pattern on a screen after passing through the sample. With this provision it becomes possible to form the images of high-order isochromates gaining the information on the optical homogeneity in the interior of bulk crystals.

The correct interpretation of the experimental pattern depends on the validity of the theory describing the shape of isochromates of different orders. Analysis of the available publications on this matter shows that even the simplest case of uniaxial crystals rests on a number of approximations resulting in uncertainties regarding to the shape of isochromates [2, 3, 9, 12-14]. Born and Wolf in their monograph [15] relied on a minimum of simplifications and arrived at a conclusion that the isochromates of uniaxial crystals correspond to curves of fourth order.
In more recent papers based on some additional approximations the authors arrive at a conclusion that isochromates are described by curves of second order $[2,3,7,9$, 12-14]. The available experimental or calculated isochromate figures are limited by two cases for which the optical axis either coincides with the normal to the crystal face or is perpendicular to it [2-4]. Exact expressions for isochromates were used in the present work for the calculation of conoscopy figures of lithium niobate and paratellurite. These crystals are widely used in acoustooptics and optoelectronics. Lithium niobate is also utilized as piezoelectric actuator for acoustooptic devices. Best possible uniformity is required to meet the demands of diffraction efficiency, spatial spectral resolution, image quality, suppression of side maxima of instrument function, etc.

In the present work we study the optical uniformity of large-size lithium niobate and paratellurite crystals by the method of laser conoscopy. Furthermore we calculate the changes of refraction indices and estimate the mechanical stresses responsible for the distortion of isochromate shape in the interior of the specimen.

\section{Isochromate equations for uniaxial crystals}

The exact expression for path difference $\Delta$, at which the waves interfere to a maximum has the form [15]:

$$
m \lambda=h\left(n_{\mathrm{e}} \cos \beta_{\mathrm{e}}-n_{\mathrm{o}} \cos \beta_{\mathrm{o}}\right),
$$

where $m$ is the maximum order, $\lambda$ is the wavelength in vacuum. The angles of refraction $\beta$ are unknown and should be determined.

For exact determination of the isochromate coordinates in the $X O Y$ plane perpendicular to the axis of conoscopic system the crystal physical coordinate system $x y z$ is transformed in accordance with Fig. 1 showing the crystal in the shape of rectangular parallelepiped, cone of rays falling onto the crystal, wave vector of the normal $\boldsymbol{m}$, wave vector $\boldsymbol{l}$ of the ray falling at an angle $\alpha$, wave vector $\boldsymbol{k}$ of the extraordinary wave refracted at an angle $\beta_{\mathrm{e}}$ in the crystal, coordinate system $X O Y$ in the 
plane of the screen with a point $(X, Y)$ belonging to some isochromate, angle $\psi$ between the normal $\boldsymbol{m}$ and crystal optical axis $z$, and projection lens with a focal length $f$. The axis $Y$ at the screen corresponds to the projection of optical axis $z$.

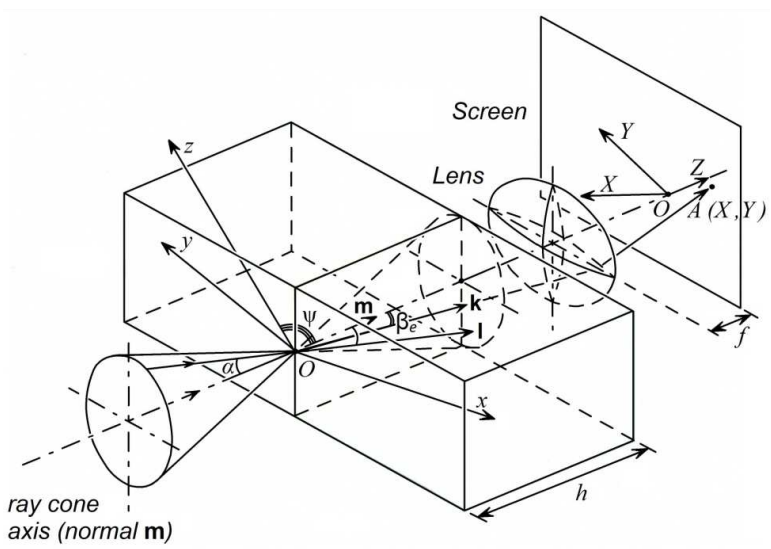

Fig. 1. Scheme of the conoscopic pattern observation. $x y z$ is the physical coordinate system of the uniaxial crystal, $X O Y$ is the coordinate system in the plane of the screen, $f$ is the focus length of the projecting lens.

The equation which may be named as equation of the conoscopic pattern of an uniaxial crystal has the following form:

$$
\begin{gathered}
\left(N_{\mathrm{o}}^{2}-N_{\mathrm{e}}^{2}\right)\left(\frac{Y \sin \psi}{\frac{m \lambda}{h} \sqrt{C}+\sqrt{N_{0}^{2} C-X^{2}-Y^{2}}}+\cos \psi\right)^{2}= \\
N_{\mathrm{o}}^{2}\left[\frac{X^{2}+Y^{2}-N_{\mathrm{e}}^{2} C}{\left(\frac{m \lambda}{h} \sqrt{C}+\sqrt{N_{\mathrm{o}}^{2} C-X^{2}-Y^{2}}\right)^{2}}+1\right],
\end{gathered}
$$

where $C=X^{2}+Y^{2}+f^{2}$.

Equation (2) is convenient for computer calculations of the isochromate shape of any order with arbitrary angles $\psi$ between the optical axis and normal to the crystal, and also for the examination of the dependence of conoscopic figures on other experimental parameters.

To finalize the theoretical question of the order of the isochromate curves for a general case of an arbitrary angle $\psi$ between the optical axis and normal, Eq. (2) should be reduced to a canonical form

$$
\begin{gathered}
B_{1} X^{8}+B_{2} X^{6} Y^{2}+B_{3} X^{6}+B_{4} X^{4} Y^{4}+B_{5} X^{4} Y^{2}+B_{6} X^{4} \\
+B_{7} X^{2} Y^{6}+B_{8} X^{2} Y^{4}+B_{9} X^{2} Y^{2}+B_{10} X^{2}+B_{11} Y^{8} \\
+B_{12} Y^{6}+B_{13} Y^{4}+B_{14} Y^{2}+B_{2} Y^{2}+B_{15}=0
\end{gathered}
$$

Thereby it is seen that in the general case the isochromates are described by the equation of eighth rather than second order. Only in some particular cases the isochromates may be described by second order curves - circles and hyperbolae. At intermediate angles $\psi$ the calculations give the isochromate shapes of the appearance different from that of second order curves. Moreover, they have different shape for different orders of $m$ and depend on the incidence angle of light $\alpha$.

\section{Some consequences of the isochromate equation}

The analysis of Eq. (2) tells us that the changes in the conoscopic patterns arising when one of the six parameters $h, A, N_{\mathrm{o}}, N_{\mathrm{e}}, f$ and $\lambda$ is varied keeping other values constant may be summarized as follows.

1. The number of observable isochromates increases whereas the distance between them decreases with the crystal thickness $h$; the number of observable isochromates and the area of the conoscopic pattern increase with the angular aperture $A$;

2. The number of isochromates increases and the distance between them decreases with the enlargement of the absolute value of the difference $\Delta N=$ $\left|N_{\mathrm{e}}-N_{\mathrm{o}}\right|$;

3. Increase of the focal length $f$ results in an increase of the distance between isochromates accompanied by an enlargement of conoscopic pattern area;

4. The distance between the isochromates increases with the light wavelength while the area of the whole pattern remains unchanged.

It follows from the above remarks that with the aid of Eq. (2) it is possible to find a combination of light aperture $A$, focus length $f$ and wavelength $\lambda$ ensuring the most informative conoscopic figure of large-size crystal of given thickness $h$ and known refractive indices $N_{\text {o }}$ and $N_{\mathrm{e}}$.

\section{Optical anomalies in lithium niobate and paratellurite single crystals}

The experimental setup for conoscopic studies included laser with a wavelength of $532 \mathrm{~nm}$, collimator, lens transforming parallel ray bundle into a cone shape, analyzer, projection lens and half-transparent frosted glass screen having a digital videocamera behind it. $\mathrm{LiNbO}_{3}$ crystals with a diameter up to $70 \mathrm{~mm}$ and paratellurite $\left(\alpha-\mathrm{TeO}_{2}\right)$ (diameter $65 \mathrm{~mm}$ ) were chosen as objects of study.

The angle between the axes $2 \mathrm{~V}$ in stressed $\mathrm{LiNbO}_{3}$ and $\mathrm{TeO}_{2}$ may be as high as $1.5-2.5^{\circ}$. Figure $2 \mathrm{a}$ and $\mathrm{b}$ demonstrates the conoscopic figures of large-size $\mathrm{LiNbO}_{3}$ and $\mathrm{TeO}_{2}$ with considerable anomalous biaxiality in the volume of the boules. The anomalous biaxiality decreases with the radius towards the central part of the crystal attaining values below 20-15' (Fig. 2c and d).

High sensitivity of the conoscopic figures to the gradient of refraction indices caused by nonuniform heating of operating elements makes it possible to observe the arising of anomalous biaxiality in uniaxial crystals. Figure 3 shows the changes in the first order isochromate in the conoscopic figure of paratellurite single crystal serving as an acousto-optic line of a working deflector.

Figure 3a corresponds to the disconnected deflector at ambient temperature of $20^{\circ} \mathrm{C}$, while Fig. $3 \mathrm{~b}$ was registered in $20 \mathrm{~s}$ after switching on the device into a working regime of power consumption $3.5 \mathrm{~W}$ at $80 \mathrm{MHz}$. 

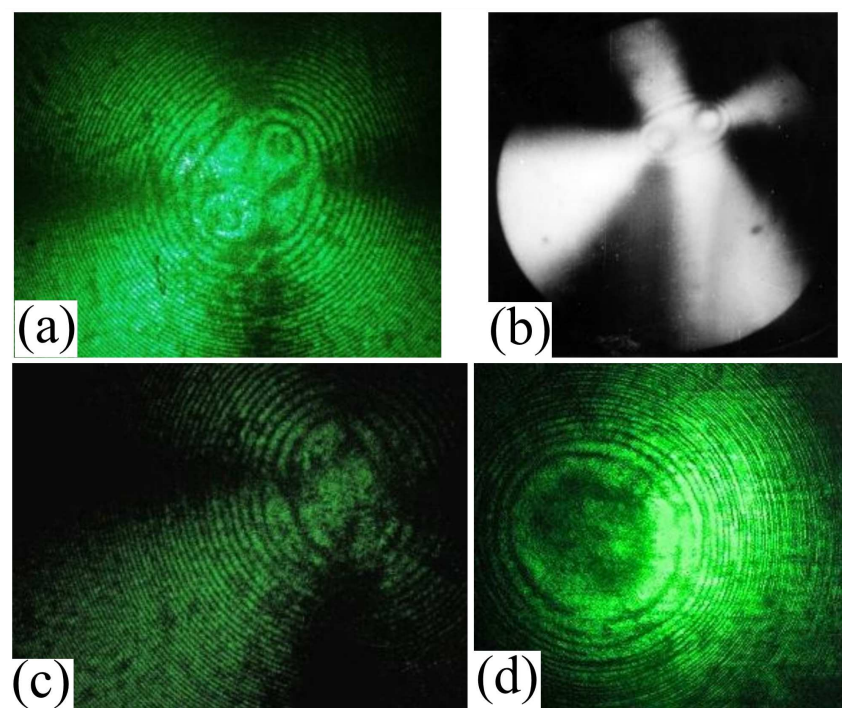

Fig. 2. Conoscopic figures of near-surface regions of large-size $\mathrm{LiNbO}_{3}$ and $\mathrm{TeO}_{2}$ crystals with anomalous biaxiality $2 V=50^{\prime}$ (a) and $2 V=1^{\circ} 20^{\prime}$ (b), respectively; (c) and (d) are the conoscopic figures for the central parts of the same samples with $2 \mathrm{~V}=20^{\prime}$ and $2 V=13^{\prime}(\mathrm{b})$, respectively.
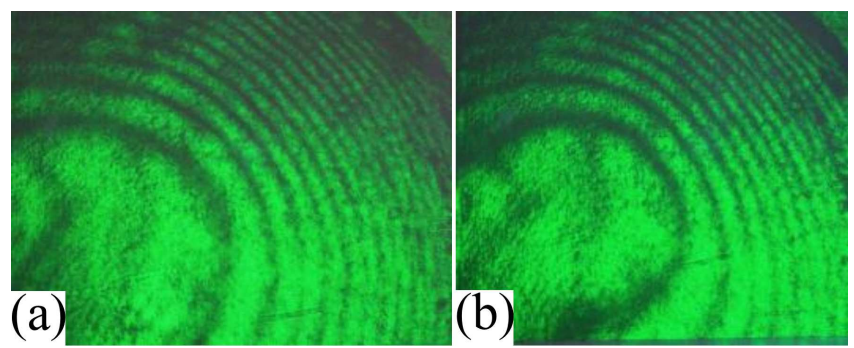

Fig. 3. Conoscopic figures of paratellurite element obtained in no-operation mode (a) and after powering the device (b).

Temperature measurements show that in this case a gradient of $\approx 5 \mathrm{~K} / \mathrm{cm}$ arises in the crystal. The heat-induced change in the position of isochromates corresponds to the changes in refraction indices as described by Eq. (2) and summary (ii) of Sect. 3 (the number of isochromates increases and the distance between them decreases with the increase of $\Delta N)$. Making the measurements of the ellipse axes with energized device and calculating the distance between the new foci it is possible to find the new angle $2 V_{1}$ between the straight lines drawn to the foci from the deflector. The difference of the angles $2 V_{1}-2 V_{0}$ should be considered as an angle $2 V_{a}$ of anomalous biaxiality due to nonuniform heating. The corresponding calculations yield a value of $2 V_{a}=43 \pm 1^{\prime}$.

The estimates of mechanical stresses $\sigma$ in the crystal volumes with anomalous biaxiality angle $2 V_{\alpha}$ between the induced axes [3] give the values of $\approx 2 \times 10^{6} \mathrm{~Pa}$ for maximal biaxiality angles and $\approx 2 \times 10^{5} \mathrm{~Pa}$ for minimal angles of biaxiality $\left(10-15^{\prime}\right)$. The experiments with some other types of AO devices have shown that even in stationary working regimes the induced optical biaxiality may be as high as $0.5-1^{\circ}$. This factor should be taken into account in the design of corresponding devices.

\section{Conclusion}

The high sensitivity of isochromates to the crystal defects and optical anomalies in uniaxial crystals suggests to use the conoscopic method for optical quality testing of acousto-optical crystals, in particular, in situ control of optical anomalies arising due to heating by external high-power lasers in addition to self-heating of the device. The presented mathematical apparatus developed during the derivation of isochromate equations makes it possible to describe the conoscopic figures and their anomalies due to refraction indices variations and mechanical stresses in real crystals.

\section{Acknowledgments}

The work was carried out with financial support in part from the Ministry of Education and Science of the RF in the framework of Program NUST "MISiS" (No. K12014-008), RFBR Research Project No. 14-07-0088 A and Federal Target Program "R\&D in the Priority Directions of Scientific Engineering Complex of Russia for 20142020", Agreement 14.574.21.0113.

\section{References}

[1] R.E. Stoiber, S.A. Morse, Microscopic Identification of Crystals, The Ronald Press Company, New York 1972.

[2] L.A. Shuvalov, A.A. Urusovskaya, I.S. Zheludev, Physical Properties of Crystals, Vol. 4, Springer, Berlin 1988.

[3] Y.I. Sirotin, M.P. Shaskolskaya, Bases of Crystal Physics, Nauka, Moscow 1975.

[4] N. Cozzella, M. Lebeau, G. Majni, N. Paone, D. Rinaldi, Nucl. Instrum. Methods Phys. Res. A 469, 331 (2001).

[5] K. Saito, S. Sugavara, J.O. Guo, A.P. Tsai, Jpn. J. Appl. Phys. 39, 5173 (2000).

[6] G.Y. Sirat, D. Psaltis, Opt. Lett. 10, 4 (1985).

[7] B.L. Van Horn, H.H. Henning, Appl. Opt. 40, 2089 (2001).

[8] O.Yu. Punin, A.G. Stuckenberg, Crystallogr. Rep. 50, 297 (2005).

[9] A. Bajor, L. Salbut, A. Szwedowski, Rev. Sci. Instrum. 69, 1476 (1998).

[10] A.I. Kolesnikov, R.M. Grechishkin, S.A. Tretiakov, V.Ya. Molchanov, A.I. Ivanova, E.I. Kaplunova, E.Yu. Vorontsova, IOP Conf. Series Mater. Sci. Eng. 49, 012037 (2013).

[11] A.F. Konstantinova, K.A. Rudoy, B.V. Nabatov, E.A. Evtushenko, V.I. Strogonov, O.Yu. Pikul, Crystallogr. Rep. 48, 823 (2003).

[12] P.H. Äyräs, A.T. Friberg, M.A. Kaivola, M.M. Salomaa, Appl. Opt. 38, 5399 (1999).

[13] T.D. Wen, Y.S. Raptis, E. Anastassakis, I.I. Lalov, A.I. Miteva, J. Appl. Phys. D Appl. Phys. 28, 2128 (1995).

[14] N. Mamedov, N. Yamamoto, Y. Shim, Y. Ninomiya, T. Takizawa, Jpn. J. Appl. Phys. 42, 5145 (2003).

[15] M. Born, E. Wolf, Principles of Optics, Oxford 1968. 